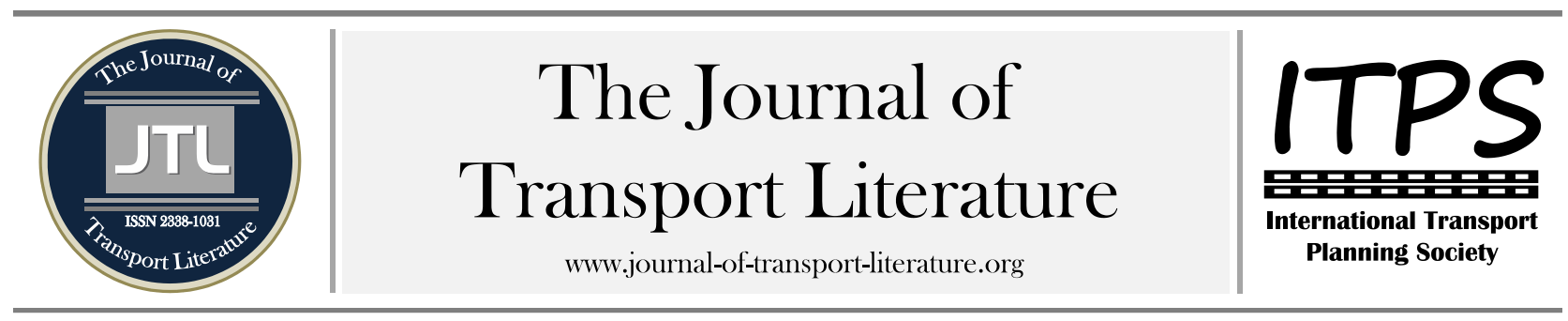

\title{
Seleção de fornecedores para atendimento às vítimas de desastres naturais considerando o lead time logístico dos produtos na fase de aquisição
}

Fabiana Santos Lima+; Daniel de Oliveira; Mirian Buss Gonçalves

Federal University of Santa Catarina, Florianópolis, Brazil

\section{Article Info}

Palavras-chave:

logística humanitária

fluxo de redes

desastre natural

Submitted 6 Dec 2013

received in revised form 18 Feb 2014; accepted 17 Jun 2014

Licensed under

Creative Commons

CC-BY 3.0 BR.

\section{Resumo}

Organizações humanitárias buscam processos eficientes e eficazes para a tomada de decisão no momento da resposta aos desastres. Evidencia-se neste estudo o uso de ferramentas logísticas para auxiliar a tomada de decisão em operações de resposta humanitária referente à coordenação de materiais para eventos em desastres naturais. Na literatura algumas abordagens têm sido propostas utilizando modelos de otimização para decisão de compras para a ajuda humanitária, sem incluir a seleção de fornecedores de forma a garantir a disponibilidade dos itens de alívio nas áreas afetadas, no momento certo. 0 presente trabalho visa desenvolver uma metodologia para definir o melhor conjunto de fornecedores respeitando, o tempo, a demanda e a capacidade de entrega no lugar atingido, desenvolvendo um modelo adaptado do problema do fluxo em redes. 0 foco desta pesquisa está nas fases da preparação e resposta, em que, com técnicas de otimização utilizada na fase da preparação, pretende-se alcançar uma resposta mais eficaz. Verificou-se que a condição de o produto estar o mais rápido possível no local de demanda foi satisfeita e foi utilizada a capacidade dos fornecedores nas datas mais próximas aos eventos com o menor custo.

+ Corresponding author. Universidade Federal de Santa Catarina. Departamento de Engenharia de Produção. Florianópolis - SC. Brasil. CEP: 88040-900.

E-mail address: fabiana.lima@posgrad.ufsc.br.

\section{Introdução}

O objetivo do presente trabalho é apresentar uma metodologia de suporte à tomada de decisão que considere o tempo de atendimento na entrega de suprimentos durante a fase de resposta aos desastres naturais, para definir o melhor conjunto de fornecedores respeitando, o tempo, a demanda e a capacidade de entrega no lugar atingido. Utiliza-se um modelo adaptado do problema do fluxo em redes. As abordagens de planejamento propostos podem ser configuradas na fase de preparação com antecedência a possíveis desastres, permitindo que as organizações humanitárias se adaptem a diferentes cenários de crise. A proposta refere-se ao processo de aquisição em operações de ajuda humanitária. 0 arcabouço teórico busca apresentar os modelos de pesquisa operacional utilizados em operações de ajuda humanitária e identifica pesquisas nas atividades de aquisição em logística humanitária. A literatura mostra que algumas abordagens têm sido propostas utilizando modelos de otimização para decisão de compras para a ajuda humanitária, mas estas abordagens não incluem a seleção de fornecedores de forma a garantir a disponibilidade dos itens de alívio nas áreas afetadas, no momento certo.

0 desenvolvimento de modelos e ferramentas que podem reduzir o tempo para a tomada de decisão, a fim de limitar o impacto dos desastres naturais é uma questão-chave. A partir deste conhecimento será possível fornecer subsídios aos órgãos responsáveis ao atendimento em desastres naturais que poderão acionar ações preventivas e emergenciais. Atividades humanitárias como compras podem ser classificadas como aquisição para a ajuda humanitária, ou seja, a ênfase principal é sobre a velocidade e disponibilidade, a fim de salvar vidas. Embora os produtos adquiridos para ajuda em operações humanitária tendem a ser itens relativamente simples, eles também podem ser bastante caros, sendo que a principal preocupação é a entrega rápida de suprimentos de alívio (Taupiac, 2001).

0 artigo esta organizado da seguinte forma: na Seção 1 é apresentada a revisão bibliográfica e, descreve alguns conceitos referentes à logística humanitária, a Seção 2 apresenta a metodologia proposta e a modelagem. 0 experimento computacional com uma pequena aplicação para o estado de Santa Catarina é apresentado na Seção 3. Considerações finais e recomendações para a continuidade dessa pesquisa são apresentadas na última seção.

\section{Pesquisa operacional e processo de aquisição em operações humanitárias}

A Logística Humanitária propõe o uso efetivo dos conceitos logísticos adaptados às especificidades da cadeia de assistência e gestão humanitária, que podem ser o diferencial para minimizar ações de improvisação e maximizar a eficiência e o tempo de resposta às situações de emergência. A coordenação de desastres envolve três fases: pré-desastre, resposta e pós-desastre. Na fase de pré-desastre ocorre à prevenção, mitigação e preparação às situações emergenciais e o desempenho da resposta ao desastre está relacionado ao nível de preparação e as metodologias utilizadas nesta fase. 0 fator resposta é uma questão-chave para a logística humanitária, tal que possibilite que o auxílio chegue o mais rápido possível, 
no lugar certo, na condição certa para ajudar vítimas de desastres (Banomyong e Sodapang, 2011).0 referencial teórico apresentado neste artigo abrange os modelos de pesquisa operacional utilizados em operações de ajuda humanitária e especifica as atividades de aquisição.

Caunhye et al (2012), analisaram modelos de otimização utilizados na logística de emergência e, a maior parte da literatura pesquisada, visa uma melhor capacidade de resposta através do uso de objetivos como, minimizar o tempo de resposta, custos de distância e demanda reprimida ao longo do tempo. A partir de uma perspectiva de cadeia de abastecimento, modelos quantitativos, existentes na literatura para auxiliar a tomada de decisão em operações humanitárias, podem ser classificados em três categorias principais: (1) aplicações de gestão de estoque, (2) localização de instalações, (3) distribuição de socorro e transporte de vítimas. A literatura de gestão de estoque (inventário) no contexto das operações de ajuda humanitária é focada em gestão de armazém, ou seja, determinar o tamanho e a frequência de pedidos, bem como os níveis de estoques de segurança, como apresentado por Beamon e Kotleba (2006). Modelos determinísticos como apresentado por Dessouky et al. (2006), Horner e Downs (2010), Jia et al. (2007), McCall (2006), Kongsomsaksakul et al. (2005) e modelos estocásticos como apresentados por Balcik e Beamon (2008), Chang et al. (2007), Duran et al. (2011), Song et al. (2009), Rawls e Turnquist (2010) determinam a localização de armazéns e centros de distribuição, no contexto do alívio humanitário. A distribuição de socorro e transporte de vítimas, especifica modelos que determinam fluxos de mercadorias ao longo de arcos. Obras de Sheu (2007), Tzeng et al. (2007) e Yan e Shih (2009) são colocados nesta categoria. Sheu (2007) desenvolveu uma pesquisa voltada a problemas de distribuição de mercadorias a partir de centros de distribuição para áreas de desastre. Dois modelos de otimização foram propostos, um para vários itens e outro para grupos homogêneos de itens. Tzeng et al. (2007) escolheram dinamicamente a quantidade de itens de alívio a serem transportados a partir de depósitos e três objetivos são alcançados: o custo total mínimo, mínimo tempo de viagem, e máxima satisfação da procura. Yan e Shih (2009) modelam o transporte de equipes de trabalho para realizar reparos de estradas no menor tempo possível. Segundo Caunhye et al (2012), modelos de alocação de recursos e fluxo de mercadorias consideram atividades que podem ser realizadas em diferentes períodos de tempo sobre os modos de transporte e commodities. Özdamar et al (2004), Yi e Kumar (2007), e Yi e Özdamar (2007) minimizam em seus modelos a soma das demandas não satisfeitas ao longo do tempo para otimizar prazos de resposta. Várias atividades voltadas ao gerenciamento da cadeia de suprimentos em organizações humanitárias, por exemplo, armazenagem, transporte e aquisição auxiliam no processo de atendimento ao desastre. 0 problema de aquisição na área de logística humanitária e gestão da cadeia de suprimentos, foi definido por Thomas e Kopczak (2005) como o processo de planejamento, implementação e controle da eficiência, relação custo-eficácia, fluxo e armazenagem de mercadorias e materiais, bem como a informação relacionada, a partir do ponto de origem até ao ponto de consumo para o propósito de melhorar o bem-estar social e aliviar a situação das pessoas mais vulneráveis (Falasca e Zobel, 2011). 0 objetivo da aquisição é assegurar que a organização humanitária tenha os recursos materiais necessários para atender aos requisitos operacionais e os requisitos de suporte operacional (Blecken, 2009). O processo de aquisição nas cadeias de abastecimento humanitário procura garantir que as organizações humanitárias têm os suprimentos necessários para atender as necessidades de alívio (PAHO em 2001, Humanitarian SupplyManagement in Logistics in the Health Sector, Pan AmericanHealth Organization, Washington, DC).As tarefas da aquisição incluem a identificação e a seleção de fornecedores adequados de forma a garantir a disponibilidade dos itens de alívio nas áreas afetadas, no momento certo. Segundo Whybark (2007) há dois aspectos para a aquisição de itens de ajuda humanitária. A primeira é a aquisição e armazenamento dos itens de alívio, em antecipação de um desastre. 0 segundo é o desenvolvimento de fontes de aquisição de itens para distribuição durante uma operação de socorro. Esta segunda atividade envolve encontrar fontes para itens comumente necessários que estejam preferencialmente localizadas perto de áreas de potenciais desastres.

Poucas abordagens quantitativas têm sido propostas sobre a aquisição em operações de ajuda humanitária. Destacamos o trabalho de Trestrail et al. (2009), que descrevem uma ferramenta de apoio à decisão MIP (mixed-integer program), a fim de melhorar o transportador marítimo e a estratégia de preços de licitação dos fornecedores de alimentos. Alternativamente, Falasca e Zobel (2011) desenvolveram modelos de decisão de compras para a ajuda humanitária. Eles usam um modelo de otimização estocástica de dois estágios para fornecer orientação em operações de socorro, a fim de minimizar a escassez de demanda esperada, bem como os custos totais de aquisição. Ambos os trabalhos visam situações específicas, uma orientação geral para o uso de métodos quantitativos em operações de socorro para auxiliar no processo de aquisição de itens de alívio não foi encontrada na literatura. Ou seja, inexistem estudos que propõem uma metodologia de suporte à tomada de decisão, que considere o tempo de atendimento na entrega de suprimentos durante a fase de resposta aos desastres naturais. A fim de auxiliar nesta lacuna de pesquisa na área de aquisições, propomos o desenvolvimento de uma ferramenta que utiliza modelos quantitativos apropriados que apoiam as tarefas de aquisição e refletem na área específica de processos em logística humanitária. A utilização desta metodologia representa a fronteira do conhecimento proposto, que se justifica na sua inovação tecnológica e científica.

A Logística Humanitária propõe o uso efetivo dos conceitos logísticos adaptados às especificidades da cadeia de assistência e gestão humanitária, que podem ser o diferencial para minimizar ações de improvisação e maximizar a eficiência e o tempo de resposta às situações de emergência (Nogueira,2010). Para isso existe a gestão de desastres que pode ser caracterizada por um ciclo dado pelas etapas de mitigação, preparação, resposta e reabilitação. Na resposta, ao contrário da logística no setor privado, as organizações humanitárias são confrontadas com o desconhecido, sem saber onde, quando e quão grande o próximo desastre será (Coppola, 2011). Na preparação, considera-se o trabalho entre desastres, o que implica estabelecer processos, mecanismos e materiais que podem ser confiáveis para responder ao ambiente dinâmico de desastres e apoiar uma resposta ágil, adaptável e alinhada. A resposta de demanda imediata necessita da mercadoria certa, na hora certa, no lugar certo e distribuído para as pessoas certas, sugerindo neste momento um sistema tipo pull. Cabe ressaltar que a metodologia proposta neste artigo é desenvolvida em função da fase de preparação e da fase de resposta conforme proposto por Charles e Lauras (2011) no Ciclo de vida da Operação Humanitária na fase 1. 


\section{Metodologia Proposta}

\subsection{Considerações iniciais}

Frente à complexidade intrínseca aos elementos envolvidos nas operações de logística humanitária é necessário adotar algumas considerações antes de apresentar a modelagem do problema: considera-se a existência de algum tipo de parceria das empresas privadas com as organizações humanitárias onde, de alguma forma, comprometam-se em informar e atualizar seus dados; utiliza-se o sistema CIF (Cost Insurance and Freight); considera-se que, enquanto a região não se encontra na situação de normalidade, qualquer tipo de ação de ajuda gera um custo. Por exemplo, as pessoas que estão desabrigadas precisam ser hospedadas em algum local como: barracas, ginásios, etc. e, quanto maior o tempo para atingir a situação de normalidade, maior o custo total do desastre. Para melhor exemplificar pode-se procurar responder à seguinte pergunta: quanto a falta deste produto custará por dia para a região atingida? Depois de esclarecidas essas considerações necessárias, busca-se definir quais serão os fornecedores que deverão ser acionados pela organização humanitária no momento da resposta, sendo primordial que os suprimentos requeridos estejam nas áreas atingidas o mais rápido possível.

\subsection{Modelagem do Problema}

0 objetivo da metodologia proposta neste trabalho é atuar dentro da fase de resposta imediata numa operação humanitária e considerando ainda que na prática o termo imediato significa atender as demandas das áreas afetadas no menor tempo possível, sob pena de vidas serem perdidas. A modelagem deve ser flexível o bastante para considerar o tempo como variável e, assim, ponderar negativamente os possíveis atrasos. Com isso, a solução caminhará de modo a escolher fornecedores que entreguem os suprimentos o mais rápido possível, mesmo que num custo mais caro, pois, do ponto de vista de vidas em risco, é a melhor opção frente a um fornecedor mais barato, mas que não conseguirá atender a demanda na data solicitada. Ou seja, o modelo deve conter algum custo de falta por dia não atendido, para que o procedimento de solução busque aqueles fornecedores de menor custo, com disponibilidade do produto no tempo solicitado, ou mais próximo disso.

Dessa forma, propõem-se um modelo de grafo onde, T é o horizonte de tempo discretizado em períodos; K é o número de fornecedores; M é o número de localidades; $F_{k}$ é o vetor com capacidade do fornecedor $\mathrm{k}$ no horizonte de planejamento $\mathrm{T} ; F_{k}=\left(F_{1 k}, F_{2 k}, \cdots, F_{T k}\right) ; k=1, \cdots, K ; D_{m}$ é o vetor com a demanda da localidade $\mathrm{m}$ no horizonte de planejamento $\mathrm{T}$ $D_{m}=\left(D_{1 m}, D_{2 m}, \cdots, D_{T m}\right) ; m=1, \cdots, M$. Têm-se dois tipos de nós: nós do tipo 1 é o par ordenado (fornecedor, tempo $)=(\mathrm{F}, \mathrm{t})$ e nós do tipo 2 é o par ordenado (localidade, tempo) $=(\mathrm{D}, \mathrm{t})$. Têm-se três tipos de arcos que representam os fluxos: arco (tipo 1), fluxos associados ao estoque $\left(\left(F_{k}, t\right),\left(F_{k}, t+1\right)\right)$, arco (tipo 2), fluxos associados à falta $\left(\left(D_{m}, t\right),\left(D_{m}, t-1\right)\right)$ e arco (tipo 3$)$, fluxos associados ao transporte $\left(\left(F_{k}, t\right),\left(D_{m}, t+\right.\right.$ tempo $\left.\left.(k, m)\right)\right)$. A Figura 1 representa o grafo considerado. 0 rótulo do nó (fornecedor, tempo) representa a quantidade de produto disponível à pronta entrega naquele dia. 0 rótulo do nó (localidade, tempo) representa a quantidade necessária do produto no local do desastre no dia solicitado. 0 objetivo é determinar quais fornecedores poderão atender ao evento de forma que o custo total de enviar o produto (suprimento) disponível através da rede seja minimizado, a fim de satisfazer a demanda dada. 0 método simplex especializado para resolver o problema de fluxo em redes, baseado na estrutura clássica em Kennington e Helgason (1980), foi então implementado. A função objetivo minimiza o custo total de transporte da provisão disponível através da rede a fim de satisfazer a demanda dada, sujeito à restrição de conservação de fluxo (Figura 2). Onde $c_{i j}$ é o custo unitário por fluxo através do arco $i \rightarrow j ; x_{i j}$ representa o fluxo através do arco $i \rightarrow j ; r_{k}$ define o fluxo líquido demandado no nó $\mathrm{k} ; u_{i j}$ é a capacidade dada no arco $i \rightarrow j$. Considera-se ainda que se $r_{k}>0$, o nó ké um nó de demanda com demanda igual a $r_{k}$; se $r_{k}<0$, o nó k é de oferta, com oferta igual a $\left|r_{k}\right|$ e, finalmente, se $r_{k}=0$, k é um ponto de transbordo.

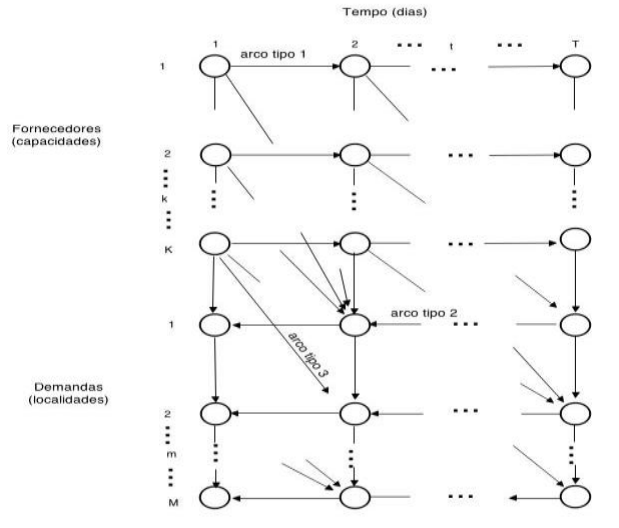

Figura 1 - Modelo do Grafo Proposto

$$
\operatorname{Min} \sum_{(i, j) \in A} c_{i j} x_{i j}
$$

s.a.

$$
\begin{array}{ll}
\sum_{(i, k) \in A} x_{i k}-\sum_{(k, j) \in A} x_{k j}=r_{k} & \forall k \in \aleph \\
0 \leq x_{i j} \leq u_{i j} & \begin{array}{l}
\text { para cada } \\
\text { arco } \mathrm{i} \rightarrow j
\end{array}
\end{array}
$$

\section{Experimento Computacional}

Considera-se, para estudo de caso, os eventos ocorridos no mês de dezembro no ano de 2011 no estado de Santa Catarina, de forma a possibilitar a adaptação de um cenário de desastre natural à metodologia proposta. No dia 13 de dezembro de 2011, no estado, ocorreram dois tipos de eventos caracterizados como desastres naturais súbitos: vendavais e granizo, que atingiram fortemente os moradores das cidades de Biguaçu, Água Doce e Balneário Barra do Sul. Segundo a Secretaria de Defesa Civil de Santa Catarina, telha é o tipo de produto emergencial mais utilizado nestes tipos de desastres, sendo assim, considerou-se este como sendo o produto necessário para o atendimento emergencial nas regiões atingidas do estudo de caso. Considerando a emergência da situação, é primordial que o produto esteja nas cidades atingidas o mais rápido possível. A Defesa Civil do estado necessita tomar a decisão referente aos fornecedores do produto, determinar aqueles fornecedores de menor custo que podem entregar o produto no tempo solicitado, ou mais próximo disso. 


\subsection{Dados de Entrada}

A Figura 3 representa os dados de entrada, com os municípios $D_{1}, D_{2}$ e $D_{3}$ sendo, respectivamente, os municípios de Água Doce, Barra do Sul e Biguaçú. Os fornecedores considerados foram $F_{1}$, localizado na cidade de São José dos Pinhais, no estado do Paraná, $F_{2}$, na cidade Barra do Sul e $F_{3}$, na cidade de São Bento do Sul, com estes dois últimos localizados em Santa Catarina. Os dados referentes à quantidade de demanda são estimados conforme a população da região atingida. Devido à principal condição ser que o produto esteja nos municípios atingidos o mais rápido possível, arbitrou-se a fim de experimentação do modelo, que a falta de cada telha por dia na região representa um custo de $R \$ 2,00$. Este valor é adicionado ao preço de cada unidade do produto a cada dia em que o produto não se encontra no local específico. A organização responsável na tomada de decisão considera que o custo de frete já está inserido no preço do fornecedor.

\section{Análise dos resultados obtidos}

Na elaboração do problema, o custo de falta do produto por dia não atendido auxilia na escolha do fornecedor no sentido de que, quando o fornecedor não tem a quantidade solicitada para aquela data, mas para outra data posterior é atribuído este custo de falta ao preço do fornecedor representado no arco do fluxo de transporte. Assim, o modelo define aqueles fornecedores com disponibilidade do produto o mais próximo do tempo solicitado e considera o menor custo total. 0 método simplex especializado para resolver o problema de fluxo em redes foi implementado utilizando a linguagem de programação Object Pascal (Mayerle, 2011). Dessa forma, o grafo resultante do modelo obteve 20 nós e 58 arcos e o método simplex desenvolvido chegou à solução após um total de 20 iterações. 0 valor total dado na solução final foi de $R \$ 60880,00$, sendo que $\mathrm{R} \$ 7400,00$ refere-se ao custo de falta e $\mathrm{R} \$ 53480,00$ ao custo do produto (custo efetivamente pago). Na representação gráfica do resultado do problema de fluxo em redes (ver Figura 4), os dados referentes à quantidade demandada são representados pelos nós de demanda na data específica e a capacidade por dia de cada fornecedor está representada nos nós do fornecedor na referente data.

Os valores ao lado dos arcos representam o quanto da capacidade do respectivo fornecedor foi utilizado no dia, por exemplo, a demanda de 5000 telhas solicitadas no dia 13 no município de Biguaçu foi atendida pelos fornecedores F1, F2 e F3 sendo que, foram utilizadas 1800 telhas do fornecedor F3 no dia 13, como esta quantidade foi enviada na data solicitada não sofreu nenhum custo de falta. Ainda no mesmo dia foram adquiridas 800 telhas do fornecedor F2 e foram 2400 telhas no dia 14 do fornecedor F1, que sofreu o custo de falta por um dia. Para atender a demanda de 2000 telhas no município de Água Doce no dia 14, foram fornecidas no mesmo dia, 1500 telhas do fornecedor F3 e 500 telhas do fornecedor F1 no dia 15, sendo neste incorporado o custo de falta por um dia. Já para atender a demanda de 800 telhas no município Barra do Sul solicitada no dia 13, foram adquiridas no dia 14, 500 telhas do fornecedor F3 e 300 telhas do fornecedor F1, considerou-se então o custo de falta por um dia. Assim, as necessidades de cada município foram atendidas e devido ao custo de falta imposto, o programa atendeu no tempo mais próximo da data de demanda, utilizando para isto, o fornecedor com capacidade suficiente para atender na data solicitada.

\begin{tabular}{|c|c|c|c|c|}
\hline & \multicolumn{3}{|c|}{ Horizonte de tempo em dias } & \\
\hline & $t_{1}=13$ & $t_{2}=14$ & $t_{3}=15$ & \\
\hline & \multicolumn{3}{|c|}{ Quantidade demandada } & \\
\hline \begin{tabular}{|l|l} 
\\
$D_{1}$
\end{tabular} & $-\cdots--$ & 2000 & ---- & \\
\hline$D_{2}$ & 800 & ---- & ---- & \\
\hline \multirow[t]{2}{*}{$D_{3}$} & 5000 & $-\cdots--$ & ---- & \\
\hline & \multicolumn{3}{|c|}{ Capacidade do fornecedor } & (R\$) p/und. \\
\hline$F_{1}$ & ---- & 2700 & 4300 & 6,4 \\
\hline $\boldsymbol{F}_{2}$ & 800 & $-\cdots--$ & ---- & 8,00 \\
\hline $\boldsymbol{F}_{3}$ & 1800 & 2000 & 2000 & 7,00 \\
\hline
\end{tabular}

Figura 3 - Dados de Entrada

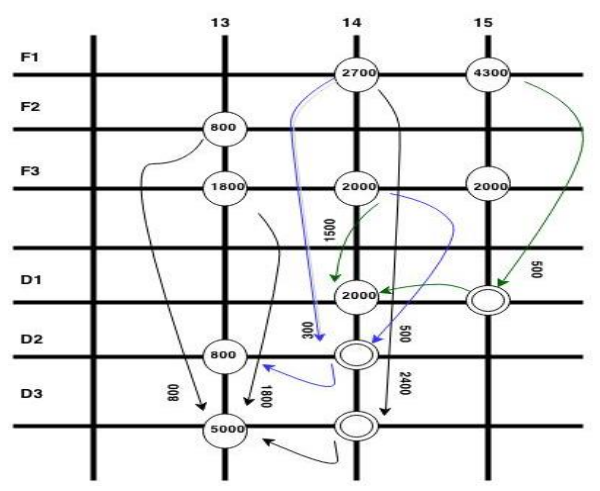

Figura 4 - Representação gráfica do resultado do problema de fluxos de rede Dados de Entrada

\section{Conclusão}

Este trabalho abordou o problema referente ao processo de aquisição em Logística Humanitária, para tanto, definiu-se o melhor conjunto de fornecedores respeitando, o tempo, a demanda e a capacidade de entrega no lugar atingido, desenvolvendo um modelo adaptado ao problema do fluxo em redes. Verificou-se que a condição de o produto estar o mais rápido possível no local de demanda foi satisfeita e foi utilizada a capacidade dos fornecedores nas datas mais próximas aos eventos com o menor custo. Foram apresentados alguns conceitos envolvidos na Logística Humanitária em desastres naturais com o objetivo de localizar o foco da pesquisa. O levantamento bibliográfico referente a modelos de pesquisa operacional e processo de aquisição em operações humanitárias demonstrou o gap de pesquisa e assim, foram abordados conceitos referentes aos problemas de fluxo em rede usado para o desenvolvimento de uma metodologia para definir o melhor conjunto de fornecedores.

Globalmente, o método calcula o custo total e é importante salientar que este custo é gerado incorporando, também, o valor do custo de falta, mas no valor final a ser efetivamente pago, este valor do índice é desconsiderado. Este custo de falta pode ser um índice que retrata o quanto a falta deste produto custa por dia para a região atingida. Isto mostra a importância do problema de fluxo em redes adaptado num contexto de Logística Humanitária em desastres naturais. Ressalta-se a importância de formar parcerias com empresas privadas, com as organizações humanitárias onde, de alguma forma, comprometam-se em informar e atualizar seus dados. A fim de entender como este modelo pode facilitar a tomada de 
decisão por diferentes indivíduos e para extrair conclusões gerais e discutir as limitações e oportunidades para apoiar a tomada de decisão em situações de emergência, está prevista a utilização de técnicas de simulação e otimização que tentam imitar o funcionamento do sistema, para estudar o impacto da aplicação do modelo na gestão dos processos de negócio na cadeia de suprimentos humanitários.

Agradecimentos

Os autores agradecem ao apoio financeiro da Capes e do CNPq.

\section{Referências}

Banomyong, R., \& Sodapang, A. (2011) Relief supply chain planning: insights from Thailand. In: Kovács, G. e Spens, K. M. (editores) Relief Supply Chain for Disasters: Humanitarian, Aid and Emergency Logistics, Helsinki: Hanken School of Economics, $31-43$.

Beamon, B. M., \& Kotleba, S. A. (2006) Inventory modelling for complex emergencies in humanitarian relief operations. International Journal of Logistics: Research and Applications, 9(1), 1-18.

Blecken, A. (2009) A Reference Task Model for Supply Chain Processes of Humanitarian Organizations (Doctorate Thesis). Institute of the University of Paderborn.

Caunhye, A. M., Nie, X., \& Pokharelet, S. (2012) Optimization models in emergency logistics: A literature review. Socio-Economic Planning Sciences, 46, 4-13.

Chang, M. S., Tseng, Y. L., \& Chen, J. W. (2007) A scenario planning approach for the flood emergency logistics preparation problem under uncertainty. Transportation Research Part E: Logistics and Transportation Review, 43(6), 737-754.

Charles, A., \& Lauras, M. (2011) An enterprise modelling approach for better optimisation modelling: application to the humanitarian relief chain coordination problem. OR Spectrum, 33(3), pp 815-841.

Coppola, D. (2011) Introduction to international disaster management. Oxford: Butterworth-Heinemann, Second edition.

Dessouky, M., Ordóñez, F., Jia, H. e Shen, Z. (2006) Rapid distribution of medical supplies. In: Hall, R. (editor) Delay management in health care systems. New York: Springer, 309-338

Duran, S., Gutierrez, M. A., \& Keskinocak, P. N. (2011) Pre-positioning of emergency items worldwide for CARE International. Interfaces, Interfaces, 41(3), 223-237.

Falasca, M., \& Zobel, C. (2011) A two-stage procurement model for humanitarian relief supply chains, Journal of Humanitarian Logistics and Supply Chain Management, 1(2), 151-169.

Horner, M. W., \& Downs, J. A. (2010) Optimizing hurricane disaster relief goods distribution: model development and application with respect to planning strategies. Disasters, 34(3), 821-844.

Jia, H., Ordóñez, F., \& Dessouky, M. (2007) Solution approaches for facility location of medical supplies for large-scale emergencies. Computers \& Industrial Engineering, 52(2), 257-276.

Kennington, J. L., \& Helgason, R.V. (1980). Algorithm for Network Programming. New York: John Willey \& Sons.

Kongsomsaksakul, S., Yang, C., \& Chen, A. (2005) Shelter location-allocation model for flood evacuation planning. Journal of the Eastern Asia Society for Transportation Studies, 6(4), 237-252.

Mayerle (2011) software "pRedeMonoProduto", não comercializável, desenvolvido pelo Prof. Dr. Sérgio Fernando Mayerle (UFSC).

McCall, V. M. (2006) Designing and prepositioning humanitarian assistance pack-up kits (HA PUKs) to support pacific fleet emergency relief operations. Master's Thesis, Department of Operations Research, Naval Postgraduate School, Monterrey, CA

Nogueira, C. W. (2010) 0 enfoque da logística humanitária na localização de uma central de inteligência e suporte para situações emergenciais e no desenvolvimento de uma rede dinâmica (Tese de Doutorado). Programa de Pós- graduação em Engenharia de Produção, Universidade Federal de Santa Catarina, Florianópolis.

Özdamar, L., Ekinci, E., \& Küçükyazici, B. (2004) Emergency logistics planning in natural disasters. Annals of Operations Research, 129, 217245.

Rawls, C. G., \& Turnquist, M. A. (2010) Pre-positioning of emergency supplies for disaster response. Transportation Research Part B: Methodological, 44(4), 521-534.

Sheu, J. B. (2007) An emergency logistics distribution approach for quick response to urgent relief demand in disasters. Transportation Research Part E: Logistics and Transportation Review, 43(6), 687-709.

Taupiac, C. (2001) Humanitarian and development procurement: a vast and growing market. International Trade Forum(4), 7-10.

Thomas, A., \& Kopczak, L. (2005) From logistics to supply chain management: The path forward in the humanitarian sector, white paper, Fritz Institute, San Francisco, CA.

Trestrail, J., Paul, J., \& Maloni, M. (2009), Improving bid pricing for humanitarian logistics, International Journal of Physical Distribution \& Logistics Management, 39(5), 428-41.

Tzeng, G. H., Cheng, H. J., \& Huang, T. D. (2007) Multi-objective optimal planning for designing relief delivery systems. Transportation Research Part E: Logistics and Transportation Review, 43(6), 673-686.

Whybark, D. C. (2007) Issues in managing disaster relief inventories. International Journal Production Economics, 108, $228-235$

Yan, S., \& Shih, Y. L. (2009) Optimal scheduling of emergency roadway repair and subsequent relief distribution. Computers \& Operations Research, 36(6), 2049-2065.

Yi, W., Kumar, A. (2007) Ant colony optimization for disaster relief operations. Transportation Research Part E: Logistics and Transportation Review, 43(6), 660-672.

Yi, W., Özdamar, L. (2007) A dynamic logistics coordination model for evacuation and support in disaster response activities. European Journal of Operational Research, 179(3), 1177-1193.

\section{Abstract (only papers in Portuguese/Spanish)}

Humanitarian organizations seeking efficient and effective processes for decision making at the time of disaster response. It is evident in this study the use of logistical tools to aid decision-making in response operations relating to the coordination of humanitarian supplies for natural disaster events. In literature some approaches have been proposed using optimization models for the purchase decision for humanitarian aid, not including the selection of suppliers to ensure the availability of relief items in the affected areas at the right time. This work aims to develop a methodology for defining the best set of suppliers respecting the time, demand and delivery capabilities in place achieved by developing a model adapted to the problem of flow in networks. The focus of this research is in preparation and response phases, where, with an optimization methodology used in preparation, intended to achieve a more effective response. It was found that the condition of the product to be as fast as possible, at the place of demand was satisfied, and was used, the ability of suppliers to the nearest event dates with the lowest cost. 